\title{
Bilateral Long Bone Fractures in a Patient with Sarcoid: A Case Report
}

\author{
Denosshan Sri ${ }^{1}$, George Tamvakopoulos ${ }^{1}$, Rosie Guy ${ }^{2}$, Adrian Butler-Manuel ${ }^{2}$ \\ ${ }^{1}$ QEII Hospital, Welwyn Garden City, UK; ${ }^{2}$ Spire Sussex Hospital, Hastings, UK. \\ Email: denosshansri@gmail.com,gst@doctors.org.uk,rosie.guy@esht.nhs.uk, adrian.buttler-manuel@esht.nhs.uk
}

Received December $26^{\text {th }}, 2012$; revised February $4^{\text {th }}, 2013$; accepted February $18^{\text {th }}, 2013$

\begin{abstract}
Although there have been many reports of small bone and vertebral involvement in sarcoidosis, long bone pathology is rare. We report a case of almost identical bilateral fractures of the proximal femoral diaphysis during separated in time through a low-energy mode of injury, and explore the difficulties encountered when seeking radiological and tissue diagnosis.
\end{abstract}

Keywords: Sarcoidosis; Osseous Sarcoidosis; Bilateral Long Bone Fractures; Alendronate; Pathological Fractures

\section{Introduction}

Sarcoidosis is a chronic systemic disease of unknown aetiology, characterised by the presence of non caseating granulomas in the organs involved. Lungs, eyes and skin are the organs most commonly affected. Osseous involvement is rare in sarcoidosis, ranging from $1 \%$ to $36 \%$, depending on the classification criteria used by previous authors. The bones reported to be most affected are the small bones of the hands and feet and are largely asymptomatic [1-7]. Long bone involvement is particularly rare.

We report a case of a long-term sarcoid patient who sustained almost identical bilateral fractures of the proximal femoral diaphysis during separate time intervals through a low-energy mode of injury.

\section{Case Report}

A 66-year-old man presented with sharp pain in his left thigh after getting up off a chair. This was in the context of a 40-year history of pulmonary sarcoidosis, for which he was on long term oral and inhaled steroids. The patient was also on alendronate for prevention of steroid induced osteoporosis. On examination he displayed cushingoid characteristics. Radiographs revealed a transverse fracture of the proximal femoral diaphysis but no evidence suggesting a pathological process to account for the low level of energy leading to the fracture. He was treated with intramedullary fixation of his fracture using a reconstruction nail and made an uncomplicated recovery (Figure 1). Reamings from his operation were sent for histology and no malignant or other disease process was identified. Bone scintigraphy revealed increased uptake at the region of his fracture site and at a similar level on his contra-lateral femur (Figure 2). Radiographs of the contra-lateral (right) femur revealed an area of cortical thickening thought to represent an area of the linea aspera (Figure 3). There was no associated periosteal thickening or other evidence to suggest pathological process. The case was reviewed in a multidisciplinary meeting with senior consultant input and was judged that preventative fixation of his contra-lateral femur was not indicated.

Four months following his original admission he represented with pain on his right leg, with identical cir-
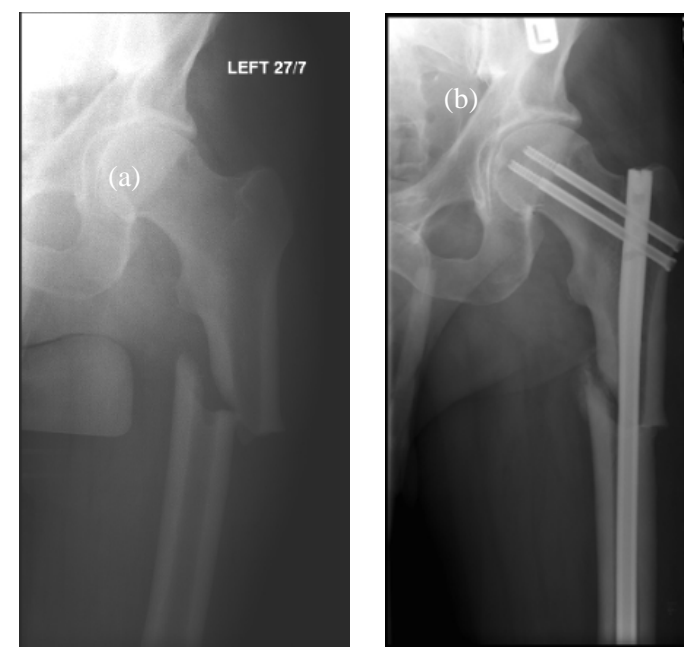

Figure 1. AP radiograph of the left hip showing proximal femoral diaphysis fracture. Pre-(a) and post-(b) IM fixation. 


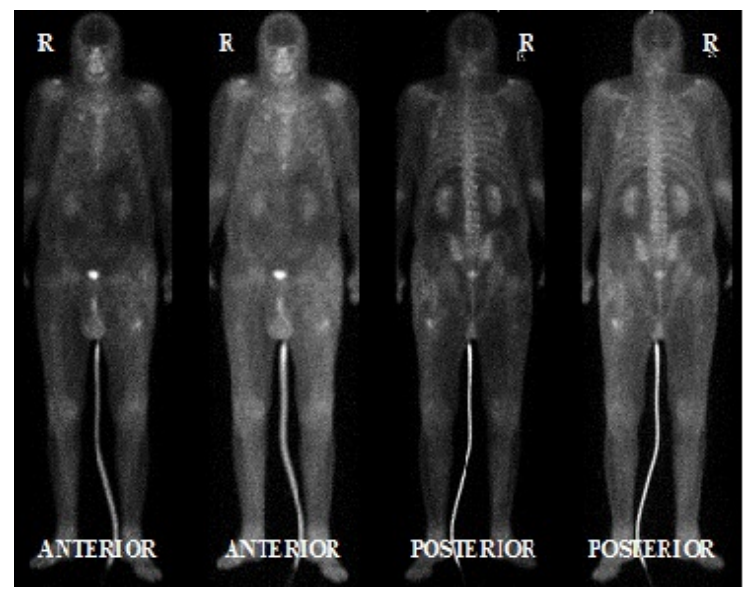

Figure 2. Bone scan revealing increased radio nucleotide uptake around the proximal femoral diaphysis bilaterally.

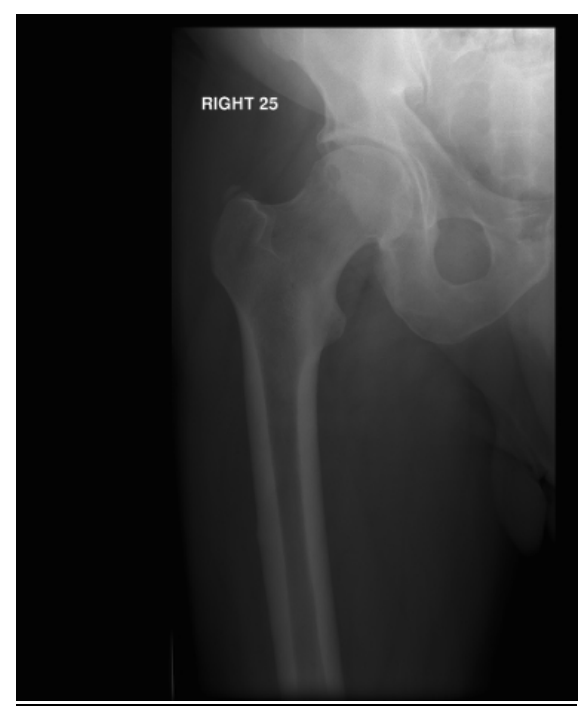

Figure 3. Radiograph of the right hip and proximal femur; suspect cortical thickening on lateral cortex of proximal diaphysis thought to represent area of linea aspera.

cumstances and mode of injury. Radiographs revealed a transverse fracture through the proximal femoral diaphysis at the level where the original abnormality was noted. He was similarly treated with fixation (Figure 4) and further reamings were sent off for histology and showed no diagnostic features.

\section{Discussion}

Osseous sarcoidosis is rare, often asymptomatic and is unusual to encounter in the absence of cutaneous lesions. The aetiology of bone involvement is unclear but it is thought that a combination of $1,25(\mathrm{OH})_{2}-\mathrm{VitD}_{3}$ mediated osteoclast activation and the release of osteoclast activating factors from local granulomas may play a role [7]. Lesions range from cystic, lytic and destructive lesions to permeative lesions whereby there is tunnelling through
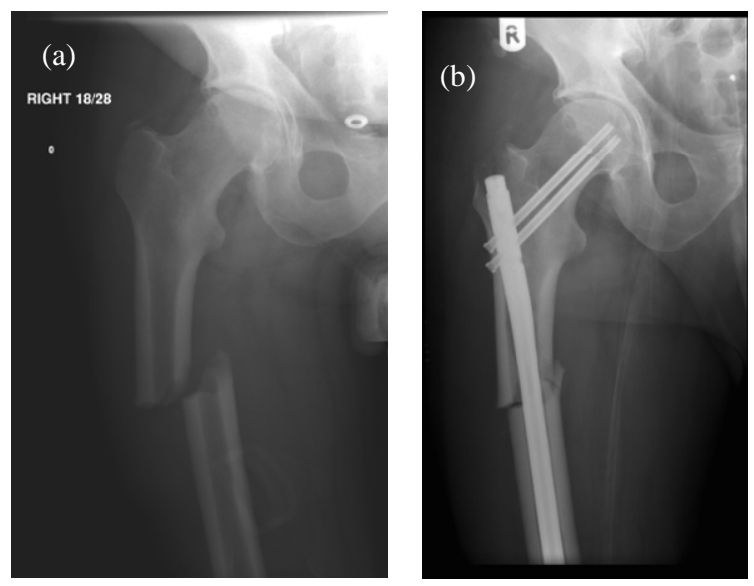

Figure 4. AP radiograph of the right hip showing an almost identical fracture occurring 4 months later; pre-(a) and post-(b) IM fixation.

the cortical and trabecular architecture. Lack of a periosteal reaction is characteristic even in diffuse involvement of the adjacent cortex [8]. It commonly affects the small bones of the hands and feet, and some cases of vertebral involvement have been reported $[1,4]$. However long bone involvement is rare; it is believed that early cases in the literature may actually have been a result of tuberculosis [5]. A pubmed search for "sarcoidosis AND long bone fractures" revealed three other cases of long bone fractures associated with sarcoidosis. Table 1 summarises the salient points from the three reports.

Corticosteroids are the drug of choice in the management of sarcoidosis, although their efficacy in osseous sarcoidosis is poor. Agents aimed at symptomatic relief range from NSAIDs and colchicine to anti-malarials and TNF inhibitors have been found to be more effective [2,5, $10,11]$. Steroids are known to contribute to a range of skeletal pathology including osteoporosis, fragility fractures and avascular necrosis [8,12]. Gonnelli et al. showed that Alendronate increased bone mineral density (BMD) by $0.8 \%$ in patients on long term steroids for sarcoid, as opposed to a $4.5 \%$ fall in BMD in the placebo treated group, whilst also reducing the rise in markers of bone resorption seen with corticosteroid use [13]. Curiously in our case bilateral long bone fractures occurred despite a bone protective regimen of alendronate.

Alendronate, an osteoclast inhibitor, is effective in the management of osteoclast driven metabolic bone disorders. Side-effects reported include gastrointestinal disturbances, whilst larger oral doses and intravenous use is linked with more serious adverse effects like renal impairment and osteonecrosis of the jaw [10]. Prolonged use of alendronate has also been reported to predispose to spontaneous fractures. Alendronate renders bone adynamic and animal studies have shown that inhibition of bone turnover results in accumulation of micro damage 
Table 1. Summary of case reports on long bone fractures in patients with sarcoidosis.

\begin{tabular}{cccccc}
\hline Study & Age, Gender & Long Bone(s) involved & Trauma status & Steroid status & Bone protection \\
\hline Redman et al., 1995 [3] & 10, Female & Distal R femur & Low energy & Nil & Not stated \\
Watson et al., 1973 [5] & 31, Female & R Olecranon & $\begin{array}{c}\text { Slipped and fell } \\
\text { onto R elbow }\end{array}$ & $\begin{array}{c}\text { 2 years 10 - 20 mg } \\
\text { Prednisolone }\end{array}$ & Not stated \\
Zickel et al., 1995 [9] & 45, Female & $\begin{array}{c}\text { Subtrochanteric R femur. } \\
\text { Subtrochanteric L femur } \\
\text { and L Ulna 5 years later }\end{array}$ & Low energy & Nil & $\begin{array}{c}\text { Calcium and Vitamin D } \\
\text { started post fractures }\end{array}$ \\
\hline
\end{tabular}

and increased susceptibility to fractures $[10,14,15]$. Femoral stress fractures in women older than 55 and on long-term bisphosphonates are 46 times higher than those not on the drug [16]. Odvina et al. looked at bone biopsies of a group of patients, which included femoral fractures, and found markedly reduced matrix and bone formation rates [15]. A retrospective study looking at femoral shaft fractures described a unicortical simple transverse fracture pattern in $76 \%$ of patients on long-term alendronate, compared to $2 \%$ in those not on the drug [14]. Confounding factors in reported cases in the literature, such as long-term corticosteroid therapy, renal impairment, metabolic bone disease, systemic disease and malignancy can skew the interpretation of the role of alendronate and bisphosphonates. Similarly this was the case in our patient. A multinational randomised double blind study demonstrated no significant difference in non-vertebral fracture incidence in patients treated with alendronate for ten years compared to placebo groups [17]. It is well established that the benefits from bisphosphonates in osteoporosis prevention outweigh the small risk of fractures reported in the literature in relation to the use of the drug.

Radiographic features of osteoporosis remain the same despite the cause, with vertebral involvement considered a hallmark. On conventional radiographs osteopenia characterised as increased lucency and cortical thinning is seen. Typically in steroid-induced osteoporosis trabecular involvement is prevalent, and the proximal femur is a commonly involved [18]. Singh described four grades of trabecular bone loss in osteoporosis, based on the radiological appearance of the trabecular trajectories in the femur [19]. Such features are not prominent in our case.

It is difficult to differentiate between sarcoid and bisphosphonate induced fractures on plain film due to fairly non-specific findings. In the acute setting bone scans reveal increased uptake at the fracture site and along a thickened lateral cortex in bisphosphonate induced fractures [20], whilst increased uptake demonstrates both active and clinically silent areas of sarcoid involvement. Biopsies from these sites of increased uptake have shown histological characteristics of sarcoidnon caseating granulomatous lesions with asteroid bodies negative for acid fast bacilli and malignancy [21,22]. Osseous sarcoidosis is however difficult to diagnose histologically [9], and this was reflected in our case. Obtaining a diagnosis from reamings is difficult because the reaming process itself is destructive, the specimen may be subject to thermal damage or the sample itself may be insufficient [23]. A retrospective study found that up to $40 \%$ of reaming samples would not lead to a causal tissue diagnosis in cases of pathological fractures [23]. Nevertheless, there have been reports in the literature of sarcoidosis and osseous involvement being diagnosed in the absence of histology [8].

Sarcoidosis is a systemic inflammatory disorder, typically affecting young adults, involving a range of organs; pulmonary being the most common [11]. It is often asymptomatic and diagnosed incidentally. Constitutional features such as weight loss, fatigue and myalgia are the most common symptoms. Cutaneous manifestations, including erythema nodosum, subcutaneous nodules and lupus pernio, and joint involvement may also be seen with shortness of breath and dry cough being the most prominent pulmonary symptoms. The course of sarcoidosis remains unpredictable with spontaneous remission on one end of the spectrum and progression to end stage lung fibrosis on the other. Corticosteroids remain the cornerstone of treatment and are used in all forms of the disease, and have been shown to improve symptoms, chest radiograph features and lung function tests [6,11, 12]. Although the long term efficacy may of our medical management strategies may be in question, early diagnosis of the condition would allow for initiation of therapy and symptom control in a typically young patient demographic.

\section{Conclusion}

We report a rare case of bilateral pathological long bone fractures in a patient with known sarcoidosis. Despite the absence of a histological diagnosis, the clinical and bone scan features combined with the rarity of bisphosphonate induced fractures are strongly suggestive of osseous sarcoidosis as the cause of bilateral identical low-energy long bone fractures. 


\section{REFERENCES}

[1] N. P. Cohen, J. Gosset, R. B. Staron and W. N. Levine, "Vertebral Sarcoidosis of the Spine in a Football Player," The American Journal of Orthopedics, Vol. 30, No. 12, 2001, pp. 875-877.

[2] S. A. Hasni, D. Kunz, K. Finzel and B. L. Gruber, "Osseous Sarcoidosis Treated with Tumor Necrosis FactorInhibitors: Case Report and Review of the Literature," Spine, Vol. 35, No. 18, 2010, pp. E904-E907.

[3] D. S. Redman, R. E. McCarthy and J. F. Jimenez, "Sarcoidosis in the Long Bones of a Child. A Case Report and Review of the Literature," The Journal of Bone \& Joint Surgery, Vol. 65, No. 7, 1983, pp. 1010-1014.

[4] L. S. Alvarez, L. M. Giner, C. A. Carbonell and J. R. C. Alborch, "Osseous Infiltration in a Patient with Sarcoidosis,” European Journal of Internal Medicine, Vol. 17, No. 5, 2006, pp. 366-367. doi:10.1016/j.ejim.2005.12.013

[5] R. C. Watson and I. Cahen, "Pathological fracture in long bone sarcoidosis. Report of a Case," The Journal of Bone \& Joint Surgery, Vol. 55, No. 3, 1973, pp. 613-617.

[6] J. A. Wiegand and M. H. Brutsche, "Sarcoidosis Is a Multisystem Disorder with Variable Prognosis-Information for Treating Physicians,” Swiss Medical Weekly, Vol. 136, No. 13-14, 2006, pp. 203-209.

[7] A. Wilcox, P. Bharadwaj and O. P. Sharma, "Bone Sarcoidosis," Current Opinion in Rheumatology, Vol. 12, No. 4, 2000, pp. 321-330. doi:10.1097/00002281-200007000-00016

[8] S. Hamoud, S. Srour, O. Fruchter, E. Vlodavsky and T. Hayek, "Lytic Bone Lesion: Presenting Finding of Sarcoidosis,” Israel Medical Association Journal, Vol. 12, No. 1, 2010, pp. 59-60.

[9] R. E. Zickel, R. S. Bernstein, S. F. Ryan and W. P. Carney, "Pathological Ununited Fractures of the Long Bones in a Patient Who Had Sarcoidosis. A Case Report," Journal of Hand Surgery, Vol. 12, No. 2, 1987, pp. 279-283.

[10] K. Cermak, F. Shumelinsky and J. Alexiou, "Case Reports: Subtrochanteric Femoral Stress Fractures after Prolonged Alendronate Therapy," Clinical Orthopaedics and Related Research, Vol. 468, No. 7, 2010, pp. 19911996. doi:10.1007/s11999-009-1192-0

[11] E. S. Chen and D. R. Moller, "Sarcoidosis-Scientific Progress and Clinical Challenges," Nature Reviews Rheumatology, Vol. 7, No. 8, 2011, pp. 457-467. doi:10.1038/nrrheum.2011.93

[12] C. J. Johns and T. M. Michele, "The Clinical Management of Sarcoidosis. A 50-Year Experience at the Johns Hopkins Hospital,” Medicine (Baltimore), Vol. 78, No. 2, 1999, pp. 65-111. doi:10.1097/00005792-199903000-00001

[13] S. Gonnelli, P. Rottoli, C. Cepollaro, C. Pondrelli, V. Ca- ppiello, M. Vagliasindi, et al., "Prevention of Corticosteroid-Induced Osteoporosis with Alendronate in Sarcoid Patients,” Calcified Tissue International, Vol. 61, No. 5, 1997, pp. 382-385. doi:10.1007/s002239900352

[14] A. S. Neviaser, J. M. Lane, B. A. Lenart, F. Edobor-Osula and D. G. Lorich, "Low-Energy Femoral Shaft Fractures Associated with Alendronate Use," Journal of Orthopaedic Trauma, Vol. 22, No. 5, 2008, pp. 346-350. doi:10.1097/BOT.0b013e318172841c

[15] C. V. Odvina, J. E. Zerwekh, D. S. Rao, N. Maalouf, F. A. Gottschalk and C. Y. Pak, "Severely Suppressed Bone Turnover: A Potential Complication of Alendronate Therapy," The Journal of Clinical Endocrinology \& Metabolism, Vol. 90, No. 3, 2005, pp. 1294-1301. doi:10.1210/jc.2004-0952

[16] J. Schilcher and P. Aspenberg, "Incidence of Stress Fractures of the Femoral Shaft in Women Treated with Bisphosphonate," Acta Orthopaedica, Vol. 80, No. 4, 2009, pp. 413-415. doi:10.3109/17453670903139914

[17] H. G. Bone, D. Hosking, J. P. Devogelaer, J. R. Tucci, R. D. Emkey, R. P. Tonino, et al., "Alendronate Phase III Osteoporosis Treatment Study Group. Ten Years' Experience with Alendronate for Osteoporosis in Postmenopausal Women," The New England Journal of Medicine, Vol. 350, No. 12, 2004, pp. 1189-1199. doi:10.1056/NEJMoa030897

[18] G. Guglielmi, S. Muscarella and A. Bazzocchi, "Integrated Imaging Approach to Osteoporosis: State-of-the-Art Review and Update," Radio Graphics, Vol. 31, No. 5, 2011, pp. 1343-1364. doi:10.1148/rg.315105712

[19] M. Singh, A. R. Nagrath and P. S. Maini, "Changes in Trabecular Pattern of the Upper End of the Femur as an Index of Osteoporosis," The Journal of Bone \& Joint Surgery, Vol. 52, No. 3, 1970, pp. 457-467.

[20] D. Mangino and D. E. Stover, "Sarcoidosis Presenting as Metastatic Bony Disease. A Case Report and Review of the Literature on Vertebral Body Sarcoidosis,” Respiration, Vol. 71, No. 3, 2004, pp. 292-294. doi:10.1159/000077430

[21] M. R. Rajebi, A. Shahrokni and M. Chaisson, "Uncommon Osseous Involvement in Multisystemic Sarcoidosis," Annals of Saudi Medicine, Vol. 29, No. 6, 2009, pp. 485486. doi:10.4103/0256-4947.57175

[22] C. Heaver and A. Marsh, "Femoral Intramedullary Biopsy: Improving Tissue Sampling," Annals of The Royal College of Surgeons of England, Vol. 93, No. 5, 2011, pp. 419420. doi:10.1308/003588411X582717g

[23] K. Hassan, S. Kalra and C. Moran, "Intramedullary Reamings for the Histological Diagnosis of Suspected Pathological Fractures,” Surgeon, Vol. 5, No. 4, 2007, pp. 202204. doi:10.1016/S1479-666X(07)80003-5 